\title{
Intimate partner violence exposure predicts antisocial behavior via pro-violence attitudes among males with elevated levels of cortisol
}

\author{
Melissa K. Peckins ${ }^{1}$ (D) | Daniel S. Shaw ${ }^{2}$ | Rebecca Waller ${ }^{1,3}$ | Luke W. \\ Hyde $^{1,4,5}$
}

${ }^{1}$ Department of Psychology, University of Michigan, Ann Arbor, Michigan

${ }^{2}$ Department of Psychology, University of Pittsburgh, Pittsburgh, Pennsylvania

${ }^{3}$ Department of Psychiatry, University of Michigan, Ann Arbor, Michigan

${ }^{4}$ Center for Human Growth and

Development, University of Michigan, Ann Arbor, Michigan

${ }^{5}$ Institute for Social Research, University of Michigan, Ann Arbor, Michigan

\section{Correspondence}

Luke W. Hyde, Department of Psychology, Center for Human Growth and Development, Institute for Social Research, University of Michigan, 1004 East Hall, 530 Church Street, Ann Arbor, MI.

Email: LukeHyde@UMich.edu

\section{Funding information}

National Institute of Mental Health, Grant/ Award Number: MH 50907 and MH 01666; National Institute of Child Health and Human Development, Grant/Award Number: 2T32 HD007109-36; National Institute on Alcohol Abuse and Alcoholism, Grant/Award Number: 2T32 AA007477-24A1t

\begin{abstract}
The present study tested whether attitudes toward violence mediate the association between intimate partner violence exposure and antisocial behavior across adolescence, and whether cortisol level moderates these pathways in an ethnically diverse sample of 190 boys from low-income, urban families. Results suggest that a pathway from intimate partner violence exposure at age 12 to antisocial behavior at age 17 is explained by pro-violence attitudes at age 15. Boys with greater exposure to intimate partner violence endorsed stronger pro-violence attitudes, which predicted increases in antisocial behavior. Further, the pro-violence attitudes to antisocial behavior pathway were stronger among boys with heightened versus dampened cortisol levels. Results suggest that violent attitudes are important for understanding the cognitive underpinnings of antisocial behavior following intimate partner violence exposure, particularly in youth with high cortisol levels. Implications for prevention and intervention are discussed with respect to targeting malleable child behavior linked to later antisocial behavior.
\end{abstract}

\section{KEYWORDS}

Adolescence, Antisocial Behavior, Attitudes, Cortisol, Violence 


\section{1 | INTRODUCTION}

Exposure to violence is a robust predictor of future antisocial behavior (AB) in youth (Margolin \& Gordis, 2000). Based on this established relationship, researchers have turned their attention to understanding the mechanisms through which specific types of exposure to violence increase risk for $A B$, with the goal of informing the development of treatments and preventive interventions that target these mechanisms (Lochman \& Wells, 2004). Research examining Social Information Processing (SIP) (Dodge, Pettit, Bates, \& Valente, 1995; Huesmann \& Guerra, 1997) suggests that cognitive factors mediate pathways from exposure to violence in the home to later AB (Dodge et al., 1995). However, beyond SIP models, little work has examined the role of attitudes toward violence among teens in promoting $A B$, particularly among youth growing up in low-income, urban environments where such attitudes may be more prevalent, accepted, and adaptive (Funk, Baldacci, Pasold, \& Baumgardner, 2004; Hyde, Shaw, \& Moilanen, 2010). Moreover, although studies suggest that both maltreatment and community violence predict $A B$ and pro-violence attitudes (Guerra, Huesmann, \& Spindler, 2003; McMahon, Felix, Halpert, \& Petropoulos, 2009), few studies have examined whether violence in the home that is not directed at the child promotes pro-violence attitudes via social learning. Finally, many children exposed to these risk factors do not show high levels of AB (Martinez-Torteya, Bogat, Von Eye, \& Levendosky, 2009), prompting the need to understand biological mechanisms that explain susceptibility to the adverse effects of violence exposure. Theories on biological sensitivity to context emphasize that youth with exaggerated biological reactivity to stress are most susceptible to harmful environmental experiences and are therefore at greater risk for developing behavior problems following exposure to violence (Belsky, BakermansKranenburg, \& Van IJzendoorn, 2007; Boyce \& Ellis, 2005; Ellis \& Boyce, 2008). However, it is not known whether biological sensitivity to context also increases vulnerability for developing $A B$ and acting on pro-violence attitudes following violence exposure in adolescence.

Pubertal onset represents a critical developmental period during which to explore how violence exposure might impact the development of $A B$. Indeed, pubertal onset marks a period of rapid increase in frequency of $A B$ particularly among boys, and when AB becomes more serious in nature (Moffitt, 1993). In addition, attitudes regarding behavior focused on aggression and violence become more stable during puberty (Huesmann \& Guerra, 1997). Finally, puberty is an important period of development for crystallization in functioning of the stress response system (Lupien, McEwen, Gunnar, \& Heim, 2009). Together, these findings suggest that violent experiences occurring during puberty may have a more marked impact on attitude formation than experiences occurring in early childhood or late adolescence. Thus, the primary goals of the current study were to: 1) Examine pro-violence attitudes as a mediator of the link between intimate partner violence (IPV) exposure and AB in the transition from early to late adolescence in a sample of boys raised in low-income, urban environments; and 2) Examine whether the link between IPV exposure and $A B$ was moderated by cortisol.

\section{1 | IPV exposure, pro-violence attitudes, and $A B$}

Numerous studies have established exposure to violence across multiple contexts during childhood and adolescence as a risk factor for AB in youth (Margolin \& Gordis, 2000; Tolan, 2016). Social learning theories have provided accounts for the effects of exposure to violence on externalizing behaviors, suggesting that youth learn by observing behaviors and their consequences (Bandura, 1978). Exposure to violence in the home that is perpetrated by a caregiver may provide a powerful learning opportunity for youth, as youth are likely to imitate the behavior of role models, a status often assigned to parents (Bricheno \& Thornton, 2007; Hurd, Zimmerman, \& Reischl, 2011). Research supports this notion, finding that youth are at greater risk of becoming a perpetrator and engaging in $A B$ more broadly after growing up in a home with high versus low levels of IPV (Ireland \& Smith, 2009; Stith et al., 2000). 
A plausible mechanism through which IPV exposure increases risk for $A B$ is the development of pro-violence attitudes. IPV exposure is thought to contribute to attitude formation in youth by reinforcing the normalcy and acceptability of violence and promoting violence as a viable problem-solving strategy (Huesmann \& Guerra, 1997). According to SIP models, these experiences lead to the incorporation of violence into youths' cognitive schemas, resulting in increases in AB (Ng-Mak, Salzinger, Feldman, \& Stueve, 2002). However, there is mixed evidence from studies that have explored links among violence exposure, pro-violence attitudes, and $A B$ around the timing of puberty. For example, violence normalization was found to be a mechanism leading to increases in AB following neighborhood and community violence exposure in an ethnically diverse sample of children ages 9-12 from urban neighborhoods (Guerra et al., 2003) and in a primarily African American sample of youth ages 10-14 recruited from low income schools in Chicago (McMahon et al., 2009). However, another study showed that exposure specifically to inter-parental aggression at age 12 was not related to pro-violence attitudes at age 12 or delinquency-related violence at ages 16 and 17 (Brendgen, Vitaro, Tremblay, \& Wanner, 2002). Thus, more research is needed to test whether proviolence attitudes explain the specific link between IPV exposure and $A B$, particularly while controlling for other co-occurring exposures to violence (i.e., community violence).

\section{2 | Elevated cortisol levels as a marker of vulnerability}

Although IPV exposure has been linked to the formation of pro-violence attitudes and AB, not all children show these attitudes following such exposures. Biological Sensitivity to Context theory suggests that biological reactivity to environmental stressors (e.g., increased heart rate) moderates the effects of environmental stressors on future health and behavior (Belsky et al., 2007; Boyce \& Ellis, 2005; Ellis \& Boyce, 2008). Specifically, youth with heightened stress reactivity are thought to be more likely to develop health and behavior problems in the context of high adversity. Previous studies have captured biological sensitivity to context by measuring physiological reactivity to stress, including heart rate, mean arterial blood pressure (MAP), respiratory sinus arrhythmia (RSA), and pre-ejection period (PEP) (Ellis, Essex, \& Boyce, 2005; Obradović, Bush, Stamperdahl, Adler, \& Boyce, 2010). An alternative, non-invasive way to measure biological sensitivity to context is to study the stress hormone cortisol, a product of hypothalamic-pituitary-adrenal (HPA) axis activation. Circulating levels of cortisol have been widely used in research to understand social and environmental influences on functioning of the stress response system and how responsivity of the stress response system relates to future health and behavior problems (Hunter, Minnis, \& Wilson, 2011; Jessop \& Turner-Cobb, 2008). For example, it is well documented that exposure to chronic and traumatic stress can lead to HPA axis dysregulation across the lifespan (De Bellis \& Zisk, 2014; Miller, Chen, \& Zhou, 2007). Previous research has established that both hyper- and hypo-activation of the HPA axis are associated with mental health outcomes (Ehlert, Gaab, \& Heinrichs, 2001; Tsigos \& Chrousos, 2002).

Cortisol output is a good candidate to index biological sensitivity to context because cortisol release is sensitive to social and environmental challenges (Dickerson \& Kemeny, 2004). In the first study to test biological sensitivity to context theory formally, Obradović et al. (2010) found an interaction between adversity and cortisol reactivity, such that 4-6-year-old children with heightened cortisol reactivity reported lower prosocial behavior in high adversity environments and higher prosocial behavior in low adversity environments (Obradović et al., 2010). This finding suggests youth with higher levels of cortisol are more sensitive to both the positive and negative aspects of the environment, faring better in low adversity environments and worse in high adversity environments compared to youth who are less biologically responsive (Belsky \& Pluess, 2009). Thus, in the context of IPV exposure, youth with higher levels of cortisol may be more receptive to IPV and therefore, more susceptible to developing and acting on pro-violence attitudes, ultimately contributing to greater $A B$. This hypothesis is consistent with a biological sensitivity to context framework, but has yet to be tested empirically. 


\section{3 | The present study}

Despite evidence implicating violence exposure within the home as an important contributor to attitudes and behavior pertaining to violence (Flood \& Pease, 2009), less is known about how exposure to IPV contributes to proviolence attitudes and broader $A B$ during adolescence in ethnically diverse samples of youth. Further, few studies have explored how youths' biological sensitivity to context influences the adoption of pro-violence attitudes and $A B$ across adolescence. In the present study, we tested a developmental pathway linking IPV exposure during early adolescence to later adolescent $A B$ via pro-violence attitudes in an ethnically diverse sample of boys from low-income families. We also examined IPV exposure during early and middle childhood to test the specificity of IPV in early adolescence as a predictor of pro-violence attitudes and $A B$ in adolescence. We hypothesized that increased exposure to IPV at age 12 would lead to a stronger pro-violence attitude at age 15 , resulting in greater frequency of $A B$ at age 17. Moreover, we expected that there would be an indirect effect from IPV to AB via pro-violence attitudes (i.e., adoption of pro-violence attitudes will explain part of the pathway from IPV to $A B$ ). We also examined whether cortisol levels during the study protocol moderated vulnerability for adopting a pro-violence attitude and engaging in $A B$ following IPV exposure. In line with biological sensitivity to context theory (Ellis \& Boyce, 2008), we expected pathways between IPV exposure and pro-violence attitudes to $A B$ to be stronger for boys with higher levels of cortisol (i.e., index of greater biological sensitivity to context). Finally, we examined these developmental pathways in boys because, compared to girls, boys are at greater risk of adopting pro-violence attitudes and engaging in $A B$ ( $A r c h e r$, 2004; Flood \& Pease, 2009; Funk, Elliott, Urman, Flores, \& Mock, 1999), and may differ in their biological response to stress (Bouma, Riese, Ormel, Verhulst, \& Oldehinkel, 2009; Kudielka \& Kirschbaum, 2005).

\section{2 | METHOD}

\section{1 | Participants}

Participants were part of the Pitt Mother \& Child Project (PMCP), a longitudinal study of child vulnerability and resiliency in low-income families (Shaw, Gilliom, Ingoldsby, \& Nagin, 2003; Shaw, Hyde, \& Brennan, 2012). Boys rather than girls were selected because of the former's higher rates of serious $A B$ in later childhood and adolescence, a primary focus of the study (Trentacosta et al., 2011). In 1991 and 1992, 310 infant boys and their mothers were recruited from Women, Infants, and Children (WIC) Nutrition Supplement Clinics when the boys were between 6 and 17 months old. At the time of recruitment, $53 \%$ of the target children in the sample were European American, 36\% were African American, 5\% were biracial, and 6\% were of other races (e.g., Hispanic American or Asian American). Two-thirds of mothers in the sample had 12 years of education or less. The mean per capita income was $\$ 241$ per month ( $\$ 2,892$ per year), and the mean Hollingshead SES score was 24.5 , indicative of a low socioeconomic standing (SES) sample. Thus, boys in this study were considered at elevated risk for $A B$ because of their SES, gender, and urbanicity, a notion that has been validated by their relatively high rate of arrests/court petitions as juveniles: $38 \%$ (Shaw et al., 2012).

Retention rates were generally high at each of the 13 time points from age 1.5-17 years of age. Ninety-94\% of the initial 310 participants completed assessments at ages 5 and 6 ; some data were available on $89 \%(n=275)$ of participants at ages 10,11 , or 12 ; and some data were available on $89 \%(n=272)$ of participants at ages 15 , 16, or 17 (Hyde et al., 2010; Shaw et al., 2012). The present study only includes a subset of families whose target children had complete cortisol data at the age 12 assessment $(n=190)$. The 190 families included in the subsample did not differ from those excluded on race/ethnicity or average monthly income $(p>0.40)$. However, mothers of target children with complete cortisol data at age 12 had a higher educational attainment and SES at the age 18-month assessment compared to families excluded from the study $(p<0.05)$. 


\section{2 | Procedures}

Target children and their families participated in 2-3-hr visits in the home and/or the lab almost yearly from age 1.5-22 years, with data collection occurring within the home at 2, 5, 5.5, 8, 10, 12, 15, and 17 years, and in the lab at 2, 3.5, 6, 11, 20, and 22 years. In addition, participants completed assessments via mail, phone, and/or online at $3,16,18,21$, and 23 years. Home assessments included structured observational tasks that are not the focus of the present report (Shaw et al., 2003). During assessments, caregivers and when sufficiently old, target children completed questionnaires regarding sociodemographic characteristics, family issues (e.g., parenting, family members' relationship quality), and child behavior. Caregivers were primarily mothers but also included fathers, stepparents, and grandparents. Participants were reimbursed for their time (Shaw et al., 2012).

\section{3 | Measures}

\subsection{1 | Intimate partner violence (IPV) exposure}

Exposure to IPV was assessed at age 12 with primary caregiver report on the Physical Aggression subscale from the Conflict Tactics Scale (Straus, 1979), a widely used parent report measure of conflict and violence in intimate relationships. The Physical Aggression subscale consists of 10 items each on a 6-point Likert scale ranging from 'Never' to 'More than 20 times'. Sample items include 'slapped the other one' and 'kicked, bit, or hit with a fist'. Items were summed to create a composite score, such that higher scores represent greater frequency of IPV exposure over the past six months at age $12(\alpha=0.78)$. At the age 12 assessment, $15.8 \%$ of the parents reported that they were not currently dating or living with anyone. However, parents still completed the Conflict Tactics Scale to capture conflict and violence in current and past relationships over the previous 6 months. Exposure to IPV was also assessed at 3.5, 6, and 10 years with the Conflict Tactics Scale (Straus, 1979) and included as a covariate in models to make sure that any effects found were of IPV during early adolescence and not the stability of this exposure since early childhood. Higher scores represent greater frequency of IPV exposure over the past year at age $3.5(\alpha=0.89)$ and past six months at age $6(\alpha=0.93)$ and $10(\alpha=0.81)$.

\subsection{2 | Pro-violence attitudes}

Pro-violence attitudes were measured at age 15 by youth reports on the Culture of Violence subscale from the Attitudes Towards Violence scale (Funk et al., 1999). The Culture of Violence subscale captures adolescents' acceptance and value of violence in the community and consists of seven items, each on a 5-point Likert scale ranging from 'Strongly Disagree' to 'Strongly Agree'. Example items include 'I could see myself committing a violent crime in 5 years' and 'It's okay to use violence to get what you want'. Items were summed and higher scores indicate stronger pro-violence attitudes. Reliability for the Culture of Violence subscale was not ideal, but acceptable, and lower in this sample of boys $(\alpha=0.66)$ than the sample of boys and girls on which this measure was normed $(\alpha=0.75)$ (Funk et al., 1999).

\subsection{3 | Antisocial behavior (AB)}

$A B$ was assessed at age 17 with the Self-Report of Delinquency (SRD) (adapted from Elliott, Huizinga, \& Ageton, 1985), a measure of the frequency with which youth engaged in AB over the past year. The SRD consists of 62 items, which youth rated on a 3-point Likert scale ranging from 'Never' to 'More often', with items summed and higher scores indicating greater frequency of $A B(\alpha=0.93)$. Sample items include 'Have you hit one of your parents' and 'Have you taken something from a store without paying for it'. AB was also assessed at age 12 with the younger version of the SRD, which consists of 26 items $(\alpha=0.82)$. 


\subsection{4 | Cortisol}

At the 12-year home-based assessment, target children provided three saliva samples during different parts of the assessment. Immediately upon arrival at the home, following consent procedures, the first saliva sample was collected (Time [T]-45 mins). Participants then completed questionnaires for 40 mins, including the Hot Topics Parent/ Child form (adapted from Hetherington et al., 1997), used to identify issues of contention between the target child and their caregiver(s) for discussion during a family interaction task. Target children then completed a 5-min relaxation protocol during which they were instructed to sit quietly and read a comic book of their choice. At the end of 5 mins, the second saliva sample was collected (T-0 mins). Following collection of the second saliva sample, target children and their caregiver(s) completed a family interaction task where they discussed 'hot topics' derived from the Hot Topics Parent/Child form for 6 mins, engaged in a parental monitoring task for 5 mins, then discussed family attitudes about substance use for $7 \mathrm{mins}$, before taking a family picture. This sequence of family tasks lasted approximately 25 mins. Participants completed questionnaires for 45 mins following completion of the family interaction task, at which time the final saliva sample was collected ( $T+70$ mins).

All saliva samples were collected between the hours of 9 AM and 3 PM (Mean interview start time = 11:13AM). Following home assessments and upon return to the laboratory, saliva samples were immediately frozen until time of assay. Saliva samples were assayed for cortisol using a high sensitivity salivary cortisol enzyme immunoassay kit (Cat. No. 1-0102/1-0112) from Salimetrics (State College, PA). For the majority of participants, raw cortisol concentrations were highest at sample $1(M=0.22 \mu \mathrm{g} / \mathrm{dL})$ and declined throughout the visit, suggesting participants were most biologically responsive to the arrival of the study team, potentially because of anticipatory stress or novelty of the situation, and less responsive to other portions of the assessment (sample $2 \mathrm{M}=0.20 \mu \mathrm{g} / \mathrm{dL}$; sample $3 \mathrm{M}=0.13 \mu \mathrm{g} / \mathrm{dL})$.

Because of our interest in youths' overall sensitivity to context, Area Under the Curve with Respect to Ground (AUCg) was calculated to measure the total secretion of cortisol across the three sampling times (Pruessner, Kirschbaum, Meinlschmid, \& Hellhammer, 2003) during the study protocol. We were particularly interested in youth with heightened levels of cortisol; therefore, we used a median split to create a heightened ( $n=95, \mathrm{MAUCg}=30.90$, $S D=13.35)$ and dampened cortisol-level group $(n=95, \mathrm{MAUCg}=12.73, S D=3.46)$. A median split allows for equalsized groupings, accounts for non-normality of the data, and utilizes the entire sample. Findings did not differ when a Mean split approach was used. In this sample, medication use was not associated cortisol concentrations at the three sampling times or cortisol AUCg.

\subsection{5 | Covariates}

To account for ethnic differences in attitudes toward violence, use of violent behavior (Funk et al., 1999), and HPA axis function (DeSantis et al., 2007), race was included in analyses by creating a dummy code for 'White' $(n=103)$ and 'Non-White' $(n=87)$ youth. To account for potentially confounding effects of exposure to neighborhood violence, which has been previously found to impact pro-violence attitudes and HPA axis function (Guerra et al., 2003; McMahon et al., 2009; Peckins, Dockray, Eckenrode, Heaton, \& Susman, 2012), neighborhood dangerousness was assessed via parent report at the age 10 assessment using the Me and My Neighborhood (Pitt Mother \& Child Project, 2001) questionnaire. The neighborhood dangerousness factor is a sum score consisting of 14 items on a 4-point Likert scale ranging from 'Never' to 'Often', with higher scores equaling greater levels of neighborhood dangerousness in the past year $(\alpha=0.90)$. Example items from the neighborhood dangerousness factor included 'a family member was robbed or mugged' and 'a family member was stabbed or shot' in the participant's neighborhood.

To account for youth hostile attribution biases, the Dodge Social Information Processing Task (Dodge \& Somberg, 1987) was administered to target youth at the 10- and 11-year assessments. Interviewers orally presented the target child with eight social vignettes and accompanying pictures. In each vignette, the behavior of another boy leads to a negative outcome for the target child (e.g., being bumped), with the intentions of the other boy left ambiguous. 
Following each vignette, the target child was asked to assess the child's attribution of intent of the 'other boy' (i.e., did the other boy hurt the target child on purpose?) and how they would respond in the situation (e.g., tell a teacher, yell at the boy). The attributions were coded 'hostile' if the target child responded that the 'other boy' performed the action on purpose. A sum score of the total number of hostile attributions was created, with higher scores indicating greater frequency of hostile attributions $(\alpha=0.64)$. Scores from the age 10 assessment were used in analyses and supplemented with scores from age 11 when missing $(n=12)$. As earlier AB could be associated with later pro-violence attitudes and AB (Moffitt, 1993), we controlled for age 12 AB in the model. Moreover, because IPV is likely to be relatively stable over time (Thompson et al., 2006), we controlled for IPV exposure at ages 3.5, 6, and 10 to assess the unique effects of this experience during the transition to adolescence.

\section{3 | RESULTS}

\subsection{Analytic plan}

Structural equation models (SEM) were examined in MPlus version 7 (Muthén \& Muthén, 2012) to test: (1) whether pro-violence attitudes at age 15 mediated the association between IPV exposure at age 12 and AB at age 17; and (2) whether overall levels of cortisol moderated these pathways using a multi-group moderated-mediation approach, comparing youth based on overall cortisol levels (heightened vs. dampened cortisol levels). Specifically, we fixed all pathways in the two groups to be equal (i.e., fully fixed model) and incrementally freed the pathways of interest, allowing them to vary between the two groups (MacKinnon, Fairchild, \& Fritz, 2007). A pathway was considered to be significantly different between youth with heightened and dampened cortisol levels when model fit significantly improved between the fully fixed model and the model with the pathway of interest freely estimated. Models accounted for child race, exposure to IPV at 3.5, 6, and 10 (each age in a separate model), neighborhood dangerousness at age 10, hostile attributions at age 10, and $A B$ at age 12 . We modeled correlations instead of direct effects between exogenous and endogenous variables that were measured at the same age (i.e., hostile attributions and neighborhood dangerousness at age 10, IPV and AB at age 12). Parameters were estimated using Maximum Likelihood Estimation with robust standard errors (MLR) and 95\% confidence intervals (CI) for indirect effects that were obtained using bias corrected bootstrapping (Dearing \& Hamilton, 2006). We estimated indirect effects using bias corrected bootstrapping rather than older methods of mediation analysis (e.g., Baron \& Kenny, 1986), because bootstrapping indirect effects is statistically more powerful and does not assume normality of the distribution of indirect effects, an assumption frequently violated in practice (Dearing \& Hamilton, 2006; Zhao, Lynch Jr, \& Chen, 2010). Significant improvement in model fit for MLR estimated models was determined using the Satorra-Bentler Scaled Difference Chi-Square Test (Bryant \& Satorra, 2012).

\subsection{Descriptive analyses}

Descriptive statistics and bivariate correlations among study variables are presented in Table 1. In line with our hypotheses, IPV exposure at age 12, pro-violence attitudes at age 15, and AB at age 17 were all positively and significantly correlated. Overall cortisol level (cortisol AUCg) at age 12 was positively correlated with neighborhood dangerousness at age 10 and IPV exposure at age 12, suggesting that youth exposed to higher levels of violence both at home and in the community had higher cortisol concentrations throughout the study protocol. Among the covariates, IPV exposure and neighborhood dangerousness at age 10 and AB at age 12 were also positively correlated with AB at age 17. 
TAB LE 1 Descriptive statistics and correlations for study variables

\begin{tabular}{|c|c|c|c|c|c|c|c|c|c|c|c|c|}
\hline & $n$ & M & (SD) & 1 & 2 & 3 & 4 & 5 & 6 & 7 & 8 & 9 \\
\hline $\begin{array}{l}1 \text { Intimate partner } \\
\text { violence }(3.5 y)\end{array}$ & 176 & 2.99 & $(5.56)$ & & & & & & & & & \\
\hline $\begin{array}{l}2 \text { Intimate partner } \\
\text { violence (6y) }\end{array}$ & 138 & 22.35 & $(5.54)$ & $0.19^{*}$ & & & & & & & & \\
\hline $\begin{array}{l}3 \text { Intimate partner } \\
\text { violence }(10 y)\end{array}$ & 133 & 1.95 & $(2.81)$ & $0.15^{* * *}$ & $0.23^{*}$ & & & & & & & \\
\hline $\begin{array}{l}4 \text { Neighborhood } \\
\text { dangerousness (10y) }\end{array}$ & 168 & 9.13 & (8.92) & $0.17^{*}$ & 0.13 & $0.34^{* *}$ & & & & & & \\
\hline $\begin{array}{l}5 \text { Hostile attributions } \\
(10 y)\end{array}$ & 186 & 4.60 & (1.95) & 0.06 & -0.12 & -0.11 & -0.06 & & & & & \\
\hline $\begin{array}{l}6 \text { Intimate partner } \\
\text { violence }(12 y)\end{array}$ & 144 & 0.69 & $(1.82)$ & 0.11 & $0.26^{* *}$ & $0.45^{* *}$ & $0.47^{* *}$ & $-0.19^{*}$ & & & & \\
\hline $7 \mathrm{AB}(12 \mathrm{y})$ & 188 & 2.48 & $(3.57)$ & $0.15^{*}$ & 0.08 & $0.26^{* *}$ & $0.26^{* *}$ & -0.06 & $0.45^{* *}$ & & & \\
\hline 8 Cortisol AUCg (12y) & 190 & 21.81 & $(13.32)$ & 0.10 & 0.05 & 0.06 & $0.17^{*}$ & 0.06 & $0.23^{* *}$ & 0.11 & & \\
\hline $\begin{array}{l}9 \text { Pro-violence attitude } \\
(15 y)\end{array}$ & 176 & 3.88 & $(3.84)$ & -0.09 & 0.07 & -0.10 & 0.11 & -0.05 & 0.33 & $0.26^{* *}$ & 0.05 & \\
\hline $10 \mathrm{AB}(17 \mathrm{y})$ & 170 & 13.18 & $(12.44)$ & 0.02 & 0.14 & $0.22^{*}$ & $0.30^{* *}$ & -0.04 & $0.30^{* *}$ & $0.43^{* *}$ & 0.06 & $0.44^{* *}$ \\
\hline
\end{tabular}

Note. $\mathrm{AB}=$ Antisocial Behavior; $\mathrm{AUCg}=$ Area Under the Curve with Respect to Ground; Maximum likelihood estimation with robust standard errors was used, resulting in the final sample size of $N=190$.

${ }^{*} p<0.05 ;{ }^{* *} p<0.01 ;{ }^{* * *} p<0.10$

\section{3 | Pro-violence attitude mediates the IPV exposure To AB association}

We fit an SEM to test whether there was an indirect effect of IPV exposure at age 12 and AB at age 17 via pro-violence attitudes at age 15 . We found significant direct pathways from greater IPV exposure at age 12 to stronger proviolence attitudes at age $15(B=0.61, S E=0.27, \beta=0.30, p<0.05)$, and from pro-violence attitudes at age 15 to more severe $A B$ at age $17(B=1.10, S E=0.32, \beta=0.33, p<0.001)$. Moreover, bias corrected bootstrapped $95 \% \mathrm{Cl}$ 's also revealed a significant indirect effect from IPV exposure to $A B$ via pro-violence attitudes (indirect estimate $=0.67$, bootstrapped $95 \% \mathrm{Cl}[0.11,1.68]$; Figure 1).

\subsection{Moderation by biological sensitivity to context (i.e., indexed via overall levels of cortisol)}

Results from the multi-group moderation model revealed group differences in the pathway between IPV exposure to $A B$ via pro-violence attitudes based on overall levels of cortisol (Figure 2). Compared to a model in which all pathways were fixed to be the same across both groups, model fit significantly improved when the pathway from pro-violence attitude at age 15 to $A B$ at age 17 was freely estimated, Satorra-Bentler Scaled Difference $\chi^{2}$ $(1)=4.50, p<0.05$, indicating that youth with higher levels of cortisol differed significantly in this pathway (i.e., cortisol level group moderated the pro-violence attitude to $A B$ pathway).

We explored each pathway in turn. First, a stronger pro-violence attitude at age 15 predicted greater $A B$ at age 17 more strongly among youth with heightened $(B=1.54, S E=0.49, \beta=0.47, p<0.01)$ versus dampened $(B=0.77, S E=0.35, \beta=0.23, p<0.05)$ levels of cortisol at age 12 . Second, consistent with our hypothesis, there 


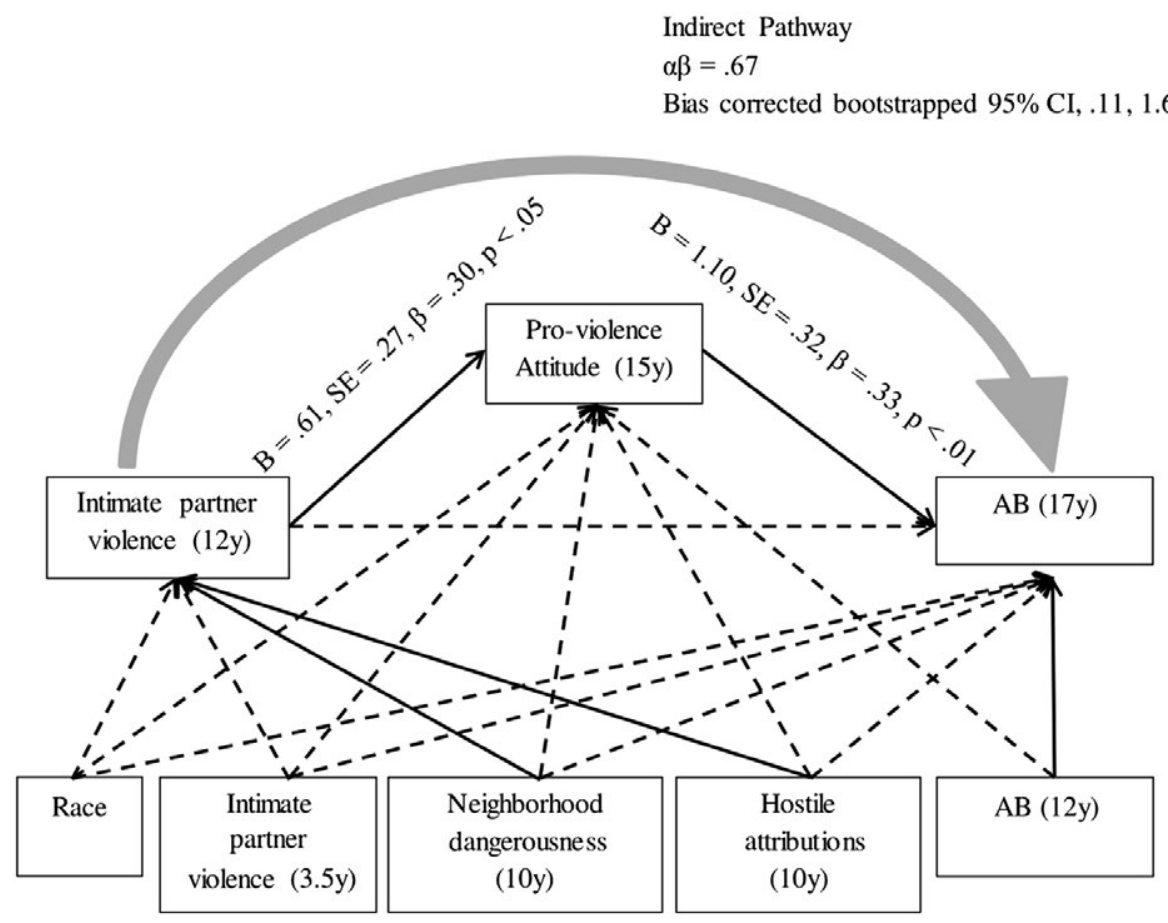

FIGURE 1 Intimate partner violence exposure contributes to greater $A B$ via stronger pro-violence attitudes Note: Dashed lines indicate non-significant pathways. Not drawn are direct paths between covariates or covariances between covariates when assessed at the same age: intimate partner violence (12y) with $A B(12 y)(B=$ 2.26, $S E=1.02, \beta=0.42, p<0.01)$ and hostile attributions $(10 y)$ with neighborhood dangerousness $(10 y)(B=-1.37$, $S E=1.17, \beta=-0.08, p>0.05)$. Average month family income (12y) was included in initial models, however, was subsequently removed due to poor model fit. Findings were consistent with and without average monthly income included as a covariate.

was a trend for greater IPV exposure at age 12 to be related to stronger pro-violence attitudes at age 15 among boys with heightened $(B=0.72, S E=0.39, \beta=0.40, p<0.10)$, but not dampened $(B=0.23, S E=0.34, \beta=0.09, n s)$ levels of cortisol at age 12. Finally, consistent with our hypothesis, the indirect pathway from IPV exposure at age 12 to $A B$ at age 17 via pro-violence attitudes at age 15 was significant only among boys with heightened (indirect estimate $=1.11$, bootstrapped $95 \% \mathrm{Cl}[0.12,3.62]$; direct estimate $=0.40$, bootstrapped $95 \% \mathrm{Cl}[-2.19,2.15])$ and not dampened (indirect estimate $=0.17$, bootstrapped $95 \% \mathrm{Cl}[-0.35,1.26]$ ) levels of cortisol at age 12 (Figure 2). However, despite the indirect pathway differing in magnitude and significance between the groups, there was no statistically significant (at $p<0.05$ ) moderation of the pathways from IPV exposure at age 12 to pro-violence attitude at age 15, or from IPV exposure at age 12 to $A B$ at age 17. Thus, cortisol level group only formally moderated the path from pro-violence attitude at age 15 to $A B$ at age 17 .

Finally, to account for exposure to violence earlier in childhood, and examine the specificity of findings for exposure to IPV during early adolescence, we tested a model that included IPV exposure at age 3.5, 6, or 10. We found a similar pattern of findings, although the indirect effect of IPV exposure at age 12 on later AB was significant only at a trend level when controlling for IPV exposure at age 6. Overall however, these additional models suggest that exposure to IPV in early adolescence (age 12) has a unique influence on attitudinal pathways to $A B$, over and above exposure to this context earlier in childhood. 


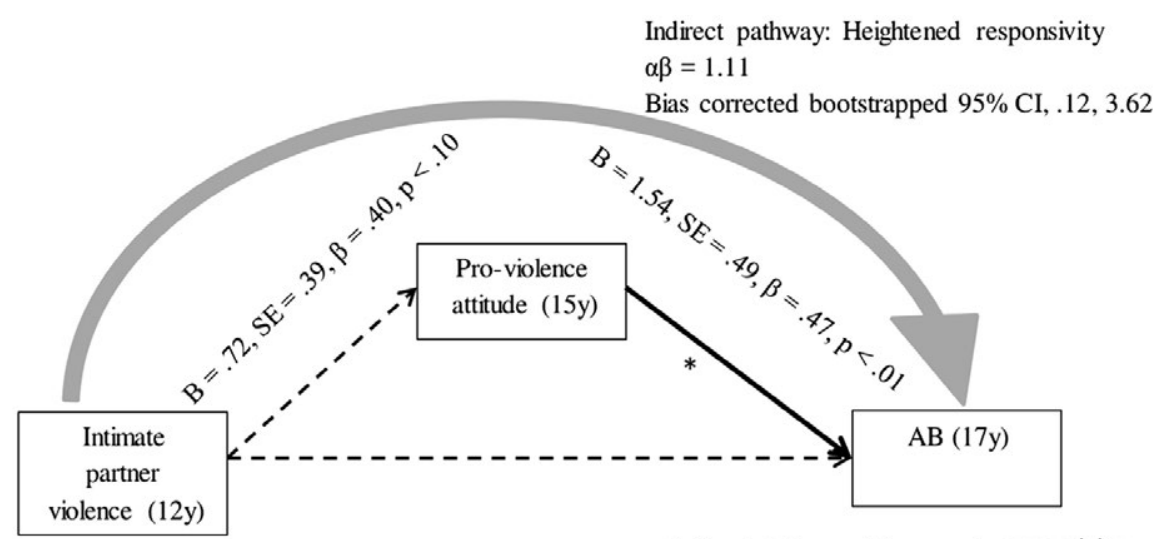

Indirect pathway: Dampened responsivity $\alpha \beta=.17$

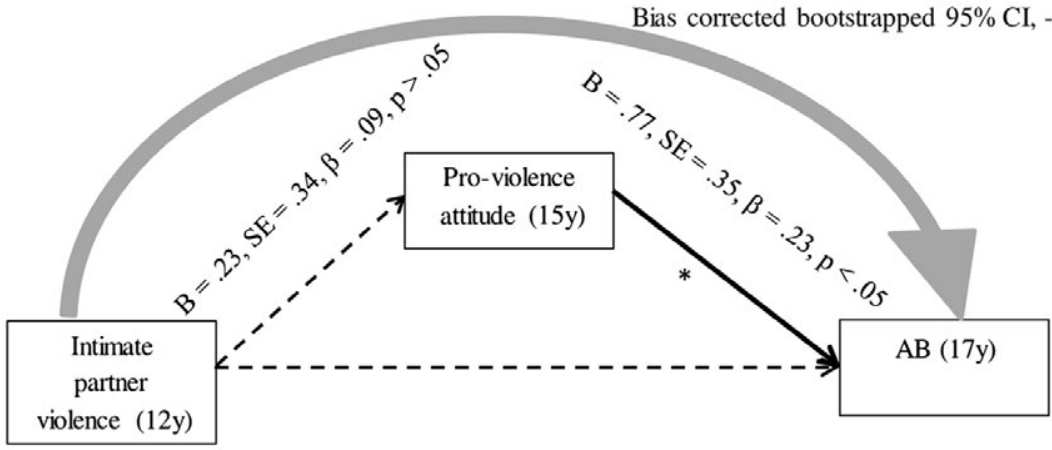

FIG URE 2 Group-based moderation-mediation model comparing boys with heightened and dampened levels of Cortisol.

Note. Solid line indicates significant pathways. Dashed lines indicate non-significant pathways. Solid, bold line and asterisk $\left(^{*}\right)$ indicates significant Satorra-Bentler Scaled $\chi^{2}$ difference test $\left(\chi^{2}=4.50, \mathrm{df}=1, p<0.05\right)$. Fully fixed model was compared to a model with the pathway from pro-violence attitude to AB freely estimated. Not drawn are pathways from and covariances with race, intimate partner violence (3.5y), neighborhood dangerousness (10y), hostile attributions (10y), and $A B(12 y)$.

\section{DISCUSSION}

The current study tested whether IPV exposure during early adolescence shapes attitudes toward violence in midadolescence and leads to greater AB in late adolescence, and whether HPA axis function moderated these pathways in an ethnically diverse sample of boys at risk for engaging in AB. Consistent with our hypotheses, we found that IPV exposure at age 12 predicted $A B$ at age 17 via pro-violence attitudes at age 15 . Furthermore, this finding was particularly strong among boys with heightened, but not dampened, levels of cortisol at age 12 . These significant direct and indirect pathways emerged even after accounting for the effects of exposure to neighborhood violence, another violence exposure and well-established risk factor for pro-violence attitudes and AB in boys (Fowler, Tompsett, Braciszewski, Jacques-Tiura, \& Baltes, 2009; Guerra et al., 2003; McMahon et al., 2009). Furthermore, we accounted for stable individual variations in hostile attributions, a component of SIP that has previously been linked to AB ( $H y d e$ et al., 2010), showing that findings were specific to pro-violence attitudes rather than simply hostile attribution biases more broadly. Finally, these results also remained when accounting for earlier exposure to IPV, suggesting a unique contribution of experiencing IPV at age 12 toward adolescent AB. Thus, our findings advance the field by identifying pro-violence attitudes as an important cognitive mechanism through which the association between IPV 
exposure and $\mathrm{AB}$ can be explained in adolescent boys. Further, this research contributes to the biological sensitivity to context literature by providing evidence that cortisol levels modulate the risk for acting on pro-violence attitudes. Our focus on the cognitive and attitudinal mechanisms is vital for extending our understanding of how AB develops and informs specific targets for youth-focused interventions to reduce AB (Galán, Choe, Forbes, \& Shaw, 2017; Galán, Shaw, Dishion, \& Wilson, 2017; Hyde et al., 2010).

\subsection{Exposure to IPV increases AB via pro-violence attitudes}

The present study demonstrated that violence experienced within the home perpetrated by caregivers, albeit not directed at the child, has an effect on $A B$ via pro-violence attitudes over and above exposure to other forms of conflict, including violence exposure in the community or neighborhood. Our results are thus consistent with extant literature and suggest that normalization and acceptability of violence are critical risk factors for the development of AB in adolescence (Guerra et al., 2003; McMahon et al., 2009), particularly when the exposure is modeled via caregiver violence. At the same time, our findings differ from those of Brendgen and colleagues (2002) who found that inter-parental aggression did not predict pro-violence attitudes and violence or specifically dating violence in adolescence. The difference in findings may be attributable to differences in measurement approaches: We assessed a broad measure of $A B$ that included violence, rule-breaking, theft, and substance use, whereas Brendgen and colleagues focused on a narrower four-item measure of physical violence perpetrated specifically in delinquency-related contexts. However, the divergence in findings among these studies emphasizes the need for further research on this topic.

\subsection{Cortisol levels and $A B$}

In line with predictions based on the biological sensitivity to context framework, we found that the effect of IPV exposure on $A B$ via pro-violence attitudes was present among youth with heightened but not dampened levels of cortisol. It is not surprising that the risk for pro-violence attitudes in relation to subsequent $A B$ was stronger for boys with heightened cortisol. Elevated endogenous cortisol concentrations and exogenously administered cortisol have been linked with increases in high-risk behavior and aggressive behavior (Böhnke, Bertsch, Kruk, Richter, \& Naumann, 2010; Putman, Antypa, Crysovergi, \& van der Does, 2009; van Bokhoven et al., 2005). Interestingly however, cortisol levels at age 12 only moderated the risk between pro-violence attitudes and future $A B$ and not the risk for developing pro-violence attitudes following IPV exposure. Although the moderating effect of cortisol on these pathways has not been tested extensively in the literature, this non-significant finding was unexpected, as cortisol levels have previously been related to learning and memory, particularly of emotionally arousing stimuli (Cahill, Gorski, \& Le, 2003; van Ast et al., 2013). Nevertheless, although sensitivity to social and environmental challenge produces a rise in cortisol concentrations, making cortisol a promising biomarker of biological sensitivity to context, it may be that other measures of biological sensitivity (e.g., RSA) (Obradović et al., 2010) or the coordination between multiple systems (Obradović \& Boyce, 2009) might be better suited to test models of biological sensitivity to context with the outcome of $A B$.

\section{3 | Strengths and limitations}

Although this study had several strengths, including the use of: an at-risk sample, a prospective longitudinal design, conservative autoregressive path models, several relevant covariates added to test specificity of pathways, and a multi-method, multi-informant measurement battery that assessed context, biology, attitudes, and behaviors, the findings should be considered within the context of several limitations. First, the sample consisted of boys from low-income families living in urban neighborhoods. Although the sample composition is a strength for establishing mechanistic pathways to $A B$ within high-risk urban boys, the findings may not generalize to girls, youth living in 
rural or suburban neighborhoods, or youth from higher SES families. Further, the present study did not address internalizing disorders that may co-occur with AB (Sareen, Stein, Cox, \& Hassard, 2004). Based on the known gender differences in the perpetration of violence, attitudes toward violence (Funk et al., 1999), and endorsement of AB and internalizing problems (Bangasser \& Valentino, 2014; Eley, Lichtenstein, \& Stevenson, 1999), future studies should test whether pro-violence attitudes also mediate the association between IPV exposure and $A B$ in a sample of girls and if these pathways are specific to $A B$ or psychopathology more broadly.

Second, the present analyses were unable to control directly for earlier HPA axis function, pro-violence attitudes, and traumatic stress, nor test the interrelationships among these biopsychosocial indices over time. For example, the HPA axis can become dysregulated in youth who have experienced chronic or traumatic stress, resulting in an elevated or attenuated cortisol response to future stressors (Trickett, Noll, Susman, Shenk, \& Putnam, 2010). Without longitudinal data on HPA axis function and pro-violence attitudes, we were unable to test how exposure to IPV shaped HPA axis responsivity over time and therefore, whether there were shifts in sensitivity to context. Future studies should test the association between IPV exposure, pro-violence attitudes, and $A B$ with data that allow for change over time to capture more fully the mechanistic process through which IPV exposure shapes attitude formation and HPA axis functioning across adolescence in youth at risk for AB.

A final limitation is in our inability to control for time of day of saliva collection and the measurement of overall concentrations of cortisol. We were unable to control for time of day in models because overall cortisol level was not modeled as a main effect but instead, included as a multi-group moderator. Further, our measure utilized three saliva samples, collected over a period of approximately $2 \mathrm{hr}$ and may have captured responsivity to the study protocol rather than reactivity to an established stress paradigm. The measure of overall cortisol levels may have also captured the normative decline in cortisol that occurs throughout the day. Thus, it is not possible to conclude from the present findings whether heightened or dampened reactivity to a well-studied acute stressor would also affect vulnerability for adopting a pro-violence attitude and engaging in AB. At the same time, previous studies have utilized similar saliva collection protocols by measuring cortisol responsivity with three samples of cortisol during a study session (Aiyer, Heinze, Miller, Stoddard, \& Zimmerman, 2014; Lee et al., 2017), with our results suggesting that a home-based assessment may be a stressor in and of itself. However, caution should be used when comparing the findings from the present study to other studies using laboratory-based stress reactivity paradigms. Thus, we suggest our findings regarding the moderating role of overall cortisol concentrations be considered as preliminary and used to guide future research on this important topic, particularly based on our strong primary finding that proviolence attitudes partially explain the risk of engaging in AB following IPV exposure during adolescence.

\section{4 | Translational implications and summary}

The study of pro-violence attitudes in adolescence offers a unique opportunity to gauge how contextual experiences such as IPV exposure shape the perception of violence as being acceptable and valued in society during a period when attitudes become more stable (Huesmann \& Guerra, 1997), and when covert and more serious AB is increasing in frequency (Moffitt, 1993). Our findings suggest a potential target for developing preventive interventions for boys exposed to IPV in early adolescence-attitudes about violence, that were found to be reliably linked to later $A B$ (Herrenkohl et al., 2000). Findings from the present study also highlight the importance of considering both cognitive and biological mechanisms through which exposure to IPV in early adolescence can be linked to $A B$ in late adolescence.

\section{ACKNOWLEDGMENTS}

The research reported in this article was supported by grants to the second author from the National Institute of Mental Health (MH 50907 \& MH 01666). The first author was supported by a National Institute of Child Health 
and Human Development T32 Fellowship in Developmental Psychology, Department of Psychology, University of Michigan (2T32 HD007109-36). The third author was supported by a National Institute on Alcohol Abuse and Alcoholism T32 Fellowship in the Addiction Center, Department of Psychiatry, University of Michigan (2T32 AA007477-24A1). The authors would like to thank Pitt Mother \& Child Project families and staff for making this research possible.

\section{CONFLICT OF INTEREST}

There are no real or perceived conflicts of interest to be reported.

\section{ORCID}

Melissa K. Peckins (iD http://orcid.org/0000-0003-2191-8614

\section{REFERENCES}

Aiyer, S. M., Heinze, J. E., Miller, A. L., Stoddard, S. A., \& Zimmerman, M. A. (2014). Exposure to violence predicting cortisol response during adolescence and early adulthood: Understanding moderating factors. Journal of Youth and Adolescence, 43, 1066-1079. https://doi.org/10.1007/s10964-014-0097-8

Archer, J. (2004). Sex differences in aggression in real-world settings: A meta-analytic review. Review of General Psychology, 8, 291-322. https://doi.org/10.1037/1089-2680.8.4.291

Bandura, A. (1978). Social learning theory of aggression. Journal of Communication, 28, 12-29.

Bangasser, D. A., \& Valentino, R. J. (2014). Sex differences in stress-related psychiatric disorders: Neurobiological perspectives. Frontiers in Neuroendocrinology, 35, 303-319. https://doi.org/10.1016/j.yfrne.2014.03.008

Baron, R. M., \& Kenny, D. A. (1986). The moderator-mediator variable distinction in social psychological research: Conceptual, strategic, and statistical considerations. Journal of Personality and Social Psychology, 51, 1173-1182. https://doi.org/10.1037/0022-3514.51.6.1173

Belsky, J., Bakermans-Kranenburg, M. J., \& Van IJzendoorn, M. H. (2007). For better and for worse: Differential susceptibility to environmental influences. Current Directions in Psychological Science, 16, 300-304. https://doi. org/10.1111/j.1467-8721.2007.00525.x

Belsky, J., \& Pluess, M. (2009). Beyond diathesis stress: Differential susceptibility to environmental influences. Psychological Bulletin, 135, 885-908. https://doi.org/10.1037/a0017376

Böhnke, R., Bertsch, K., Kruk, M. R., Richter, S., \& Naumann, E. (2010). Exogenous cortisol enhances aggressive behavior in females, but not in males. Psychoneuroendocrinology, 35, 1034-1044. https://doi.org/10.1016/j. psyneuen.2010.01.004

Bouma, E. M. C., Riese, H., Ormel, J., Verhulst, F. C., \& Oldehinkel, A. J. (2009). Adolescents' cortisol responses to awakening and social stress; Effects of gender, menstrual phase and oral contraceptives. The TRAILS study. Psychoneuroendocrinology, 34, 884-893. https://doi.org/10.1016/j.psyneuen.2009.01.003

Boyce, W. T., \& Ellis, B. J. (2005). Biological sensitivity to context: I. An evolutionary-developmental theory of the origins and functions of stress reactivity. Development and Psychopathology, 17, 271-301. https://doi.org/10.101 7050954579405050145

Brendgen, M., Vitaro, F., Tremblay, R. E., \& Wanner, B. (2002). Parent and peer effects on delinquency-related violence and dating violence: A test of two mediational models. Social Development, 11, 225-244. https://doi. org/10.1111/1467-9507.00196

Bricheno, P., \& Thornton, M. (2007). Role model, hero or champion? Children's views concerning role models. Educational Research, 49, 383-396. https://doi.org/10.1080/00131880701717230

Bryant, F. B., \& Satorra, A. (2012). Principles and practice of scaled difference chi-square testing. Structural Equation Modeling: A Multidisciplinary Journal, 19, 372-398. https://doi.org/10.1080/10705511.2012.687671

Cahill, L., Gorski, L., \& Le, K. (2003). Enhanced human memory consolidation with post-learning stress: Interaction with the degree of arousal at encoding. Learning \& Memory, 10, 270-274. https://doi.org/10.1101/Im.62403

De Bellis, M. D., \& Zisk, A. (2014). The biological effects of childhood trauma. Child and Adolescent Psychiatric Clinics of North America, 23, 185-222. https://doi.org/10.1016/j.chc.2014.01.002 
Dearing, E., \& Hamilton, L. C. (2006). V. Contemporary advances and classic advice for analyzing mediating and moderating variables. Monographs of the Society for Research in Child Development, 71, 88-104. https://doi. org/10.1111/j.1540-5834.2006.00406.x

DeSantis, A. S., Adam, E. K., Doane, L. D., Mineka, S., Zinbarg, R. E., \& Craske, M. G. (2007). Racial/ethnic differences in cortisol diurnal rhythms in a community sample of adolescents. Journal of Adolescent Health, 41, 3-13. https://doi. org/10.1016/j.jadohealth.2007.03.006

Dickerson, S. S., \& Kemeny, M. E. (2004). Acute stressors and cortisol responses: A theoretical integration and synthesis of laboratory research. Psychological Bulletin, 130, 355-391. https://doi.org/10.1037/0033-2909.130.3.355

Dodge, K. A., Pettit, G. S., Bates, J. E., \& Valente, E. (1995). Social information-processing patterns partially mediate the effect of early physical abuse on later conduct problems. Journal of Abnormal Psychology, 104, 632-643. https://doi. org/10.1037/0021-843X.104.4.632

Dodge, K. A., \& Somberg, D. R. (1987). Hostile attributional biases among aggressive boys are exacerbated under conditions of threats to the self. Child Development, 58, 213-224. https://doi.org/10.2307/1130303

Ehlert, U., Gaab, J., \& Heinrichs, M. (2001). Psychoneuroendocrinological contributions to the etiology of depression, posttraumatic stress disorder, and stress-related bodily disorders: The role of the hypothalamus-pituitary-adrenal axis. Biological Psychology, 57, 141-152. https://doi.org/10.1016/S0301-0511(01)00092-8

Eley, T. C., Lichtenstein, P., \& Stevenson, J. (1999). Sex differences in the etiology of aggressive and nonaggressive antisocial behavior: Results from two twin studies. Child Development, 70, 155-168. https://doi.org/10.1111/1467-8624.00012

Elliott, D. S., Huizinga, D., \& Ageton, S. S. (1985). Explaining delinquency and drug use. Beverly Hills, CA: Sage Publications.

Ellis, B. J., \& Boyce, W. T. (2008). Biological sensitivity to context. Current Directions in Psychological Science, 17, $183-187$. https://doi.org/10.1111/j.1467-8721.2008.00571.x

Ellis, B. J., Essex, M. J., \& Boyce, W. T. (2005). Biological sensitivity to context:Il. Empirical explorations of an evolutionary-developmental theory. Development and Psychopathology, 17, 303-328. https://doi.org/10.10170S0954579405050157

Flood, M., \& Pease, B. (2009). Factors influencing attitudes to violence against women. Trauma, Violence, \& Abuse, 10, 125-142. https://doi.org/10.1177/1524838009334131

Fowler, P. J., Tompsett, C. J., Braciszewski, J. M., Jacques-Tiura, A. J., \& Baltes, B. B. (2009). Community violence: A meta-analysis on the effect of exposure and mental health outcomes of children and adolescents. Development and Psychopathology, 21, 227-259. https://doi.org/10.1017/S0954579409000145

Funk, J. B., Baldacci, H. B., Pasold, T., \& Baumgardner, J. (2004). Violence exposure in real-life, video games, television, movies, and the internet: Is there desensitization? Journal of Adolescence, 27, 23-39. https://doi.org/10.1016/j. adolescence.2003.10.005

Funk, J. B., Elliott, R., Urman, M. L., Flores, G. T., \& Mock, R. M. (1999). The attitudes towards violence scale: A measure for adolescents. Journal of Interpersonal Violence, 14, 1123-1136. https://doi.org/10.1177/088626099014011001

Galán, C. A., Choe, D. E., Forbes, E. E., \& Shaw, D. S. (2017). The interaction between monoamine oxidase A and punitive discipline in the development of antisocial behavior: Mediation by maladaptive social information processing. Development and Psychopathology, 29, 1235-1252. https://doi.org/10.1017/S0954579416001279

Galán, C. A., Shaw, D. S., Dishion, T. J., \& Wilson, M. N. (2017). Neighborhood deprivation during early childhood and conduct problems in middle childhood: Mediation by aggressive response generation. Journal of Abnormal Child Psychology, 45, 935-946. https://doi.org/10.1007/s10802-016-0209-x

Guerra, N. G., Huesmann, L. R., \& Spindler, A. (2003). Community violence exposure, social cognition, and aggression among urban elementary school children. Child Development, 74, 1561-1576. https://doi.org/10.1111/1467-8624.00623

Herrenkohl, T. I., Maguin, E., Hill, K. G., Hawkins, J. D., Abbott, R. D., \& Catalano, R. F. (2000). Developmental risk factors for youth violence. Journal of Adolescent Health, 26, 176-186. https://doi.org/10.1016/S1054-139X(99)00065-8

Hetherington, E. M., Clingempeel, W. G., Anderson, E. R., Deal, J. E., Hagan, M. S., Hollier, E. A., \& Lindner, M. S. (1992). Coping with marital transitions: A family systems perspective [Monograph]. Monographs of the Society for Research in Child Development, 57(Serial No. 227), 2-3. doi:https://doi.org/10.1111/j.1540-5834.1992.tb00300.x

Huesmann, L. R., \& Guerra, N. G. (1997). Children's normative beliefs about aggression and aggressive behavior. Journal of Personality and Social Psychology, 72, 408-419. https://doi.org/10.1037/0022-3514.72.2.408

Hunter, A. L., Minnis, H., \& Wilson, P. (2011). Altered stress responses in children exposed to early adversity: A systematic review of salivary cortisol studies. Stress, 14, 614-626. https://doi.org/10.3109/10253890.2011.577848

Hurd, N. M., Zimmerman, M. A., \& Reischl, T. M. (2011). Role model behavior and youth violence: A study of positive and negative effects. The Journal of Early Adolescence, 31, 323-354. https://doi.org/10.1177/0272431610363160

Hyde, L. W., Shaw, D. S., \& Moilanen, K. L. (2010). Developmental precursors of moral disengagement and the role of moral disengagement in the development of antisocial behavior. Journal of Abnormal Child Psychology, 38, $197-209$. https://doi.org/10.1007/s10802-009-9358-5 
Ireland, T. O., \& Smith, C. A. (2009). Living in partner-violent families: Developmental links to antisocial behavior and relationship violence. Journal of Youth and Adolescence, 38, 323-339. https://doi.org/10.1007/s10964-008-9347-y10.1007/ s10964-008-9347-y

Jessop, D. S., \& Turner-Cobb, J. M. (2008). Measurement and meaning of salivary cortisol: A focus on health and disease in children. Stress, 11, 1-14. https://doi.org/10.1080/10253890701365527

Kudielka, B. M., \& Kirschbaum, C. (2005). Sex differences in HPA axis responses to stress: A review. Biological Psychology, 69, 113-132. https://doi.org/10.1016/j.biopsycho.2004.11.009

Lee, D. B., Peckins, M. K., Heinze, J. E., Miller, A. L., Assari, S., \& Zimmerman, M. A. (2017). Psychological pathways from racial discrimination to cortisol in African American males and females. Journal of Behavioral Medicine, 41, $208-220$. https://doi.org/10.1007/s10865-017-9887-2

Lochman, J. E., \& Wells, K. C. (2004). The coping power program for preadolescent aggressive boys and their parents: Outcome effects at the 1-year follow-up. Journal of Consulting and Clinical Psychology, 72, 571-578. https://doi. org/10.1037/0022-006X.72.4.571

Lupien, S. J., McEwen, B. S., Gunnar, M. R., \& Heim, C. (2009). Effects of stress throughout the lifespan on the brain, behaviour and cognition. Nature Reviews Neuroscience, 10, 434-445. https://doi.org/10.1038/nrn2639

MacKinnon, D. P., Fairchild, A. J., \& Fritz, M. S. (2007). Mediation analysis. Annual Review of Psychology, 58, 593-614. https://doi.org/10.1146/annurev.psych.58.110405.085542

Margolin, G., \& Gordis, E. B. (2000). The effects of family and community violence on children. Annual Review of Psychology, 51, 445-479. https://doi.org/10.1146/annurev.psych.51.1.445

Martinez-Torteya, C., Bogat, G. A., Von Eye, A., \& Levendosky, A. A. (2009). Resilience among children exposed to domestic violence: The role of risk and protective factors. Child Development, 80, 562-577. https://doi. org/10.1111/j.1467-8624.2009.01279.x

McMahon, S. D., Felix, E. D., Halpert, J. A., \& Petropoulos, L. A. (2009). Community violence exposure and aggression among urban adolescents: Testing a cognitive mediator model. Journal of Community Psychology, 37, 895-910. https://doi.org/10.1002/jcop.20339

Miller, G. E., Chen, E., \& Zhou, E. S. (2007). If it goes up, must it come down? Chronic stress and the hypothalamic-pituitaryadrenocortical axis in humans. Psychological Bulletin, 133, 25-45. https://doi.org/10.1037/0033-2909.133.1.25

Moffitt, T. E. (1993). Adolescence-limited and life-course-persistent antisocial behavior: A developmental taxonomy. Psychological Review, 100, 674-701. https://doi.org/10.1037/0033-295X.100.4.674

Muthén, L. K., \& Muthén, B. O. (2012). Mplus Version 7 user's guide. Los Angeles, CA: Muthén \& Muthén.

Ng-Mak, D. S., Salzinger, S., Feldman, R., \& Stueve, A. (2002). Normalization of violence among inner-city youth: A formulation for research. American Journal of Orthopsychiatry, 72, 92-101. https://doi.org/10.1037/0002-9432.72.1.92

Obradović, J., \& Boyce, W. T. (2009). Individual differences in behavioral, physiological, and genetic sensitivities to contexts: Implications for development and adaptation. Developmental Neuroscience, 31, 300-308. https://doi. org/10.1159/000216541

Obradović, J., Bush, N. R., Stamperdahl, J., Adler, N. E., \& Boyce, W. T. (2010). Biological sensitivity to context: The interactive effects of stress reactivity and family adversity on socioemotional behavior and school readiness. Child Development, 81, 270-289. https://doi.org/10.1111/j.1467-8624.2009.01394.x

Peckins, M. K., Dockray, S., Eckenrode, J. L., Heaton, J., \& Susman, E. J. (2012). The longitudinal impact of exposure to violence on cortisol reactivity in adolescents. Journal of Adolescent Health, 51, 366-372. https://doi.org/10.1016/j. jadohealth.2012.01.005

Pitt Mother and Child Project. (2001). Me and My Neighborhood Questionnaire. Pittsburgh, PA: University of Pittsburgh.

Pruessner, J. C., Kirschbaum, C., Meinlschmid, G., \& Hellhammer, D. H. (2003). Two formulas for computation of the area under the curve represent measures of total hormone concentration versus time-dependent change. Psychoneuroendocrinology, 28, 916-931. https://doi.org/10.1016/S0306-4530(02)00108-7

Putman, P., Antypa, N., Crysovergi, P., \& van der Does, W. A. J. (2009). Exogenous cortisol acutely influences motivated decision making in healthy young men. Psychopharmacology (Berl), 208, 257-263. https://doi.org/10.1007/ s00213-009-1725-y

Sareen, J., Stein, M. B., Cox, B. J., \& Hassard, S. T. (2004). Understanding comorbidity of anxiety disorders with antisocial behavior: Findings from two large community surveys. The Journal of Nervous and Mental Disease, 192, $178-186$. https://doi.org/10.1097/01.nmd.0000116460.25110.9f

Shaw, D. S., Gilliom, M., Ingoldsby, E. M., \& Nagin, D. S. (2003). Trajectories leading to school-age conduct problems. Developmental Psychology, 39, 189-200. https://doi.org/10.1037/0012-1649.39.2.189

Shaw, D. S., Hyde, L. W., \& Brennan, L. M. (2012). Early predictors of boys' antisocial trajectories. Development and Psychopathology, 24, 871-888. https://doi.org/10.1017/S0954579412000429

Stith, S. M., Rosen, K. H., Middleton, K. A., Busch, A. L., Lundeberg, K., \& Carlton, R. P. (2000). The intergenerational transmission of spouse abuse: A meta-analysis. Journal of Marriage and Family, 62, 640-654. 
Straus, M. A. (1979). Measuring intrafamily conflict and violence: The conflict tactics (CT) scales. Journal of Marriage and the Family, 41, 75-88. https://doi.org/10.2307/351733

Thompson, R. S., Bonomi, A. E., Anderson, M., Reid, R. J., Dimer, J. A., Carrell, D., \& Rivara, F. P. (2006). Intimate partner violence: Prevalence, types, and chronicity in adult women. American Journal of Preventive Medicine, 30, $447-457$. https://doi.org/10.1016/j.amepre.2006.01.016

Tolan, P. H. (2016). Community violence exposure and developmental psychopathology. In D. Cicchetti (Ed.), Developmental Psychopathology, Risk, Resilience, and Intervention (pp. 43-85). Hoboken, NJ: John Wiley \& Sons Inc.

Trentacosta, C. J., Criss, M. M., Shaw, D. S., Lacourse, E., Hyde, L. W., \& Dishion, T. J. (2011). Antecedents and outcomes of joint trajectories of mother-son conflict and warmth during middle childhood and adolescence. Child Development, 82, 1676-1690. https://doi.org/10.1111/j.1467-8624.2011.01626.x

Trickett, P. K., Noll, J. G., Susman, E. J., Shenk, C. E., \& Putnam, F. W. (2010). Attenuation of cortisol across development for victims of sexual abuse. Development and Psychopathology, 22, 165-175. https://doi.org/10.1017/ S0954579409990332

Tsigos, C., \& Chrousos, G. P. (2002). Hypothalamic-pituitary-adrenal axis, neuroendocrine factors and stress. Journal of Psychosomatic Research, 53, 865-871. https://doi.org/10.1016/S0022-3999(02)00429-4

van Ast, V. A., Cornelisse, S., Marin, M.-F., Ackermann, S., Garfinkel, S. N., \& Abercrombie, H. C. (2013). Modulatory mechanisms of cortisol effects on emotional learning and memory: Novel perspectives. Psychoneuroendocrinology, 38, 18741882. https://doi.org/10.1016/j.psyneuen.2013.06.012

van Bokhoven, I., Van Goozen, S. H., van Engeland, H., Schaal, B., Arseneault, L., Seguin, J. R., ... Tremblay, R. E., (2005). Salivary cortisol and aggression in a population-based longitudinal study of adolescent males. Journal of Neural Transmission, 112, 1083-1096. https://doi.org/10.1007/s00702-004-0253-5

Zhao, X., Lynch, J. G. Jr, \& Chen, Q. (2010). Reconsidering Baron and Kenny: Myths and truths about mediation analysis. Journal of Consumer Research, 37, 197-206. https://doi.org/10.1086/651257

How to cite this article: Peckins MK, Shaw DS, Waller R, Hyde LW. Intimate partner violence exposure predicts antisocial behavior via pro-violence attitudes among males with elevated levels of cortisol. Soc. Dev.. 2018;27:761-776. https://doi.org/10.1111/sode.12313 